\title{
Density functional theory study of the capacitance of single file ions in a narrow cylinder
}

\author{
Xian Kong ${ }^{a}$, Jianzhong $\mathrm{Wu}^{a}$, Douglas Henderson ${ }^{b}$
}

November 1, 2014

\author{
${ }^{a}$ Department of Chemical and Environmental Engineering, University of California, Riverside \\ CA 92521, USA \\ ${ }^{b}$ Department of Chemistry and Biochemistry, Brigham Young University, Provo UT 84602, \\ USA
}

\begin{abstract}
The differential capacitance of a model organic electrolyte in a cylindrical pore that is so narrow that the ions can form only a single file is studied by means of density functional theory (DFT). Kornyshev, Faraday Discuss. 164 (2013) 117-133, has studied this system and found the differential capacitance to have only a double hump shape (the so-called camel shape) whereas other geometries show this behavior only at low ionic concentrations that are typical for aqueous electrolytes. However, his calculation is rather approximate. In this DFT study we find that the double hump shape occurs only at low ionic concentrations. At high concentrations, the capacitance has only a single hump. Kornyshev considers a metallic cylinder and approximately includes the contributions of electrostatic images. Electrostatic images are not easily incorporated into DFT. As a result, images are not considered in this study and the question of whether Kornyshev's result is due to his approximations or images cannot be answered. Simulations to answer this question are planned.
\end{abstract}

Keywords: Single file nanopore, cylindrical double layer, capacitance, density functional theory

It is a pleasure for us to dedicate this article to Darsh Wasan, who has served so well as the Editor-in-Chief of this journal and, at the same time, has had a distinguished career as 
a university administrator, and as an outstanding teacher and research scientist. At times, we are inclined to believe that there is a set of identical triplets, all posing to be one person. In any case, one or three, Darsh Wasan is a fine human being and good friend. We are glad that he is lightening his load.

\section{Introduction}

Supercapacitors based on electric double layer expansion show great potential as an energy storage device due to their large power density, high capacitance, and sustainable life cycles $[1,2]$. Recent developments in the nanostructured electrode materials [3, 4] largely improved the performance of the supercapacitors due to their high specific surface area. In these materials, the characteristic sizes of the pores are comparable to the size of the electrolytes, namely, only single file or single layer of electrolyte ions may enter the pores.

Kornyshev [5] has published an intriguing article in which he studied the dependence of the capacitance of the double layer formed by a system of organic electrolyte ions in a cylindrical pore that is so narrow that the ions can form only a single row. This geometry is shown in Fig.1. We consider first the case where all the ions have the same diameter but do report some results for differing diameters. In the equal diameter case, to have a single file, the cylinder diameter, $D=2 a$, and ion diameter, $d$, must obey $d \leq 2 a \leq 2 d$. The system is open in the sense that the ionic concentration inside the pore is determined by that the concentration of ions in a reservoir with which the ions in the cylinder are in equilibrium. In this article, when we give a value for the concentration of the organic electrolyte, it is to be understood that this is the concentration of the organic electrolyte ions in the reservoir. Kornyshev devised an interesting Ising-like lattice theory to describe this system. The Ising theory rests upon a lattice with nearest neighbor interactions and a binary proposition (occupied or unoccupied sites or a similar proposition). It is a bit of a stretch to apply this to a fluid with long range coulombic interactions but it is not an impossiblity. Additionally, he considers a metallic cylinder wall and takes into account, albeit very approximately, electrostatic images. According to his theory, the differential capacitance of this system as a function of the surface potential always has the shape of two humps, at least for the range of parameters that he studied. The two hump shape is often referred to as a "camel" shape. However, this is not a fully satisfactory term since only bactrian camels have two humps; dromedary camels have a single hump. We find it more satisfactory to describe the shape of the capacitance curve as having a single-hump 
(or a bell) shape or a double-hump shape. Kornyshev's result is interesting because it contrasts with experience with single-surface double layers using modified Poisson-Boltzmann (MPB) theory [6], Monte Carlo (MC) simulations[7], and density functional theory (DFT) [8]. Earlier theoretical studies all show that the capacitance has a two-hump shape at only low ionic concentrations but that, as the concentration increases, the minimum between the two humps fills in and becomes a maximum. The capacitance has a bell shape or single-hump shape at higher concentrations. The question naturally arises whether the persistent double hump capacitance curves predicted by Kornshev is a feature of his Ising-like approximation or of image effects or a feature of the single file geometry. Thus, it seems worthwhile to examine this single file cylindrical ion system by means of a more realistic approximation to determine whether single-hump as well as double-hump capacitance curves could be found for single file ions in a cylindrical pore.

\section{Model}

We consider a cylindrical pore of diameter $D=2 a$ containing ions that are in equilibrium with a reservoir. The ions are treated as charged hard spheres. The diameter and charge of the cations are $d_{+}$and $Z_{+} e$, and those corresponding to anions are $d_{-}$and $Z_{-} e$, with $e$ being the magnitude of the elementary charge and $Z_{i}$ is the ion valence. There are no solvent molecules in our model. The model is appropriate for an organic electrolyte with the solvent represented by a dielectric contiuum.

For a sufficiently long cylinder $(L>>D)$ such that the end effects are negligible, the ionic density profiles, i.e., ionic distributions, can be specified by a radial coordinate $r$. The separation of a pair of ions in the cylinder is given by the difference in their radial positions, $r_{i j}$, axial positions, $z_{i j}$, and polar angles, $\theta_{i j}$. Since the ions are confined to the cylinder, the radial position of each ion must be less than $a$. The distance between the centers of the ions is denoted by $R$. The electrostatic interaction between the ions is given by

$$
\beta U_{i j}(R)=l_{B} \frac{Z_{i} Z_{j}}{R}
$$

where $\beta=1 /\left(k_{B} T\right), k_{B}$ being the Boltzmann constant and $T$ is the temperature $(300 K)$. In Eq.(1), $l_{B}$ is the Bjerrum length, defined by

$$
l_{B}=\frac{e^{2}}{4 \pi \varepsilon \varepsilon_{0} k_{B} T}
$$



tric constant, which we assumed to be 6.5 for a typical organic electrolyte. The resulting Bjerrum length is $8.55 \mathrm{~nm}$. We have also considered other values of the Bjerrum length and concentration than those presented in this study. The behavior of the $C_{d}-\psi_{0}$ curve for these different values of $l_{B}$ and concentrations is similar. For conciseness, these numerical results are not shown.

In our DFT calculations, the cylinder wall is maintained at a constant electrical potential, $\psi_{0}$. The cylinder is sufficiently narrow so that the ions form a single file, i.e., $d_{i} \leq 2 a \leq d_{i}$. The pore confinement effect modeled as a hard-wall potential. In other words, the nonCoulomb external potential due to the presense of the wall is given by

$$
V_{i}(r)=\left\{\begin{array}{l}
0, r \leq a-d_{i} / 2 \\
\infty, r>a-d_{i} / 2 .
\end{array}\right.
$$

In our DFT calculations, we assume that the cylinder surface and the region outside the cylinder have the same dielectric constant as the interior of the cylinder. Thus, electrostatic images are not considered here. Kornyshev does consider images for a metallic cylinder wall. However, his treatment of images is rather approximate. The expression that he uses for the interaction between the ions presumes that each ion lies on the central axis of the cylinder. Although this appears to be a good approximation for a single ion pore, we cannot be sure how satisfactory it is given that the cylinder surface is also not far from the central axis and that the ions are also close to the cylinder surface and images. Also, the Ising-like theory used by Kornyshev is a further approximation. In any case, image effects are not easily incorporated into DFT [9]. In view of this, we feel it best make a study of image effects in the future using simulations or the modified Poisson-Boltzmann (MPB) equation [10], where images can be systemmatically incorporated.

\section{Density functional theory}

Density functional theory (DFT) is arguably the best theory for calculating the density profiles of particles near a wall. This is especially true for ions near an electrode. We have reported our DFT study of various ionic liquids near an electrode [8, 11, 12]. For model ionic liquids, the agreement with simulations is outstanding. 


\section{Ionic distribution functions}

In the cylindrical geometry that we employ, Possion's equation for the ionic distributions is

$$
\frac{1}{r} \frac{\partial}{\partial r}\left(r \frac{\partial \psi(r)}{\partial r}\right)=-\frac{e}{\epsilon_{0} \epsilon} \sum_{i} Z_{i} \rho_{i}(r)
$$

and the basic DFT expression for $\rho(r)$ is

$$
\rho_{i}(r)=\rho_{i}^{0} \exp \left[-\beta Z_{i} e \psi(r)-\beta \Delta \mu_{i}^{e x}(r)\right],
$$

where $\rho_{i}(r)$ is the number density distribution function for the ions of species $i, \rho_{i}^{0}$ is the bulk number density, or concentration, of this species. The quantity $\mu_{i}^{e x}$ is the local excess

chemical potential of species i. An explicit expression for $\mu_{i}^{e x}$ will be given in the following subsection. The boundary conditions for Poisson's equation are

$$
\psi^{\prime}(0)=0
$$

and

$$
\psi(a)=\psi_{0}
$$

where $\psi_{0}$ is the electric potential of the surface of the cylinder.

In our DFT calculation, we start with an initial guess for the density profile that is assumed to be uniform. The electric potential, $\psi(r)$ is calculated from Eq. (4) together with the above boundary conditions, Eqs. (6) and (7). With this new $\psi(r)$, we obtain a new set of ionic profiles. A Picard iteration is used and the iteration continues until the sum over the grid points of the differences between the old and new values of $\rho_{i}$ is less than $10^{-6}$. To ensure that the convergence is stable, we mix only a smallfraction of the new profiles with the old profiles to form the next iterate.

The surface charge density on the cylinder surface is given by

$$
Q=-2 \pi e \sum_{i} Z_{i} \int_{0}^{a} \rho_{i}(r) r d r .
$$

Thus, the DFT calculation leads to a one-to-one relationship between the surface charge density, $Q$, and the surface potential, $\psi_{0}$. From this the differential capacitance is obtained from 


$$
C_{D}=\frac{\partial Q}{\partial \psi_{0}}
$$

\section{Local excess chemical potentials}

Density functional theory requires an analytical expression for $\mu_{i}^{e x}$ in Eq. (5). For the primitive model electrolyte (charged hard sphere ions and solvent represented by a continuum), that is used in this work, $\mu_{i}^{e x}$ includes contributions due to hard sphere (HS) repulsion and electrostatic correlations (EL),

$$
\mu_{i}^{e x}(\mathbf{r})=\mu_{i}^{H S}(\mathbf{r})+\mu_{i}^{E L}(\mathbf{r})
$$

The hard sphere term accounts for ionic volume exclusion effects, which can be described accurately by the modified fundamental measure theory (MFMT) [13, 14], which gives

$$
\beta \mu_{i}^{H S}(\mathbf{r})=\sum_{\alpha} \int d \mathbf{r}^{\prime} \phi_{\alpha} \omega_{i}^{\alpha}\left(\mathbf{r}-\mathbf{r}^{\prime}\right) .
$$

In Eq. (11) the summation uses six weight functions, $\omega_{i}^{\alpha}(\mathbf{r})$ that are the same as those in the original fundamental measure theory [15]. Among them, two scalar weight functions are related to the volume and surface area of a spherical particle of diameter $d_{i}$ :

$$
\omega_{i}^{(3)}(\mathbf{r})=\theta\left(r-d_{i} / 2\right)
$$

where $r=|\mathbf{r}|, \theta(r)$ is the Heaviside step function,

$$
\omega_{i}^{(2)}(\mathbf{r})=\delta\left(r-d_{i} / 2\right)
$$

and $\delta$ is the Dirac delta function. Another weight function is related to the variance across the particle surface,

$$
\omega_{i}^{(v 2)}(\mathbf{r})=\frac{\mathbf{r}}{r} \delta\left(r-d_{i} / 2\right)
$$

The other weight functions are

$$
\omega_{i}^{(0)}(\mathbf{r})=\omega_{i}^{(2)}(\mathbf{r}) /\left(\pi d_{i}^{2}\right)
$$




$$
\omega_{i}^{(1)}(\mathbf{r})=\omega_{i}^{(2)}(\mathbf{r}) /\left(2 \pi d_{i}\right)
$$

and

$$
\omega_{i}^{(v 1)}(\mathbf{r})=\omega_{i}^{(v 2)}(\mathbf{r}) /\left(2 \pi d_{i}\right)
$$

The coefficients on the right hand side of Eq. (11) are defined by the functional derivatives of the local excess Helmholtz energy density with respect to the six weighted densities:

$$
\begin{gathered}
\phi_{0}=-\ln \left(1-n_{3}\right), \\
\phi_{1}=\frac{n_{2}}{1-n_{3}}, \\
\phi_{2}=\frac{n_{1}}{1-n_{3}}+\frac{n_{3}+\left(1-n_{3}\right)^{2} \ln \left(1-n_{3}\right)}{12 \pi n_{3}^{2}\left(1-n_{3}\right)^{2}} n_{2}^{2}\left(1-\varsigma^{2}-\varsigma^{4}+\varsigma^{6}\right), \\
\phi_{3}=\frac{n_{0}}{1-n_{3}}+\frac{n_{1} n_{2}}{\left(1-n_{3}\right)^{2}}\left(1-\varsigma^{2}\right)-\frac{n_{2}^{3}\left(1-\varsigma^{2}\right)^{3}}{36 \pi}\left[\frac{2-5 n_{3}+n_{3}^{2}}{\left(1-n_{3}\right)^{3} n_{3}^{2}}+\frac{2 \ln \left(1-n_{3}\right)}{n_{3}^{3}}\right], \\
\phi_{v 1}=-\frac{\mathbf{n}_{v 2}}{1-n_{3}},
\end{gathered}
$$

and

$$
\phi_{v 2}=-\frac{\mathbf{n}_{v 1}}{1-n_{3}}-\frac{n_{3}+\left(1-n_{3}\right)^{2} \ln \left(1-n_{3}\right)}{6 \pi n_{3}^{2}\left(1-n_{3}\right)^{2}} n_{2} \mathbf{n}_{v 2}\left(1-\varsigma^{2}\right)^{2},
$$

where $\varsigma=\mathbf{n}_{v 2} / n_{2}$, and

$$
n_{\alpha}(\mathbf{r})=\sum_{i} n_{\alpha, i}(\mathbf{r})=\sum_{i} \int d \mathbf{r}^{\prime} \rho_{i}\left(\mathbf{r}^{\prime}\right) \omega_{i}^{(\alpha)}\left(\mathbf{r}-\mathbf{r}^{\prime}\right) .
$$

In the cylindrical geometry used here, the ionic density profiles vary only in the radial direction, viz, $\rho_{i}(\mathbf{r})=\rho_{i}(r)$. For this case, the weighted densities are

$$
n_{0, i}(r)=\frac{n_{2, i}(r)}{\pi d_{i}^{2}}
$$




$$
n_{1, i}(r)=\frac{n_{2, i}(r)}{2 \pi d_{i}}
$$

and

$$
n_{2, i}(r)=\frac{d_{i}^{2}}{4} \int_{0}^{2 \pi} d \varphi \int_{0}^{\pi} d \theta\left[\rho_{i}\left(r^{\prime}\right) \sin \theta\right],
$$

where $r^{\prime 2}=r^{2}+r d_{i} \cos \theta+\frac{d_{i}^{2}}{4}-\frac{d_{i}^{2}}{4} \sin ^{2} \theta \cos ^{2} \varphi$,

$$
n_{3, i}(r)=\pi \int_{0}^{d_{i} / 2} r_{1}^{2} d r_{1} \int_{0}^{2 \pi} d \varphi \int_{0}^{\pi} d \theta\left[\rho_{i}\left(r^{\prime}\right) \sin \theta\right],
$$

where $r^{\prime 2}=r^{2}+2 r r_{1}^{2} \cos \theta+r_{1}^{2}-r_{1}^{2} \sin ^{2} \theta \cos ^{2} \varphi$,

$$
\begin{gathered}
\mathbf{n}_{v 1, i}(r)=\frac{\mathbf{n}_{v 2, i}(r)}{2 \pi d_{i}}, \\
\mathbf{n}_{v 2, i}(r)=-\frac{\mathbf{r}}{r} \frac{d_{i}^{2}}{4} \int_{0}^{2 \pi} d \varphi \int_{0}^{\pi} d \theta\left[\rho_{i}\left(r^{\prime}\right) \sin \theta \cos \theta\right],
\end{gathered}
$$

where $r^{\prime 2}=r^{2}+r d_{i} \cos \theta+\frac{d_{i}^{2}}{4}-\frac{d_{i}^{2}}{4} \sin ^{2} \theta \cos ^{2} \varphi$.

Similarily, the local excess chemical potentials are

$$
\begin{gathered}
\phi_{0, i}(r)=\frac{\phi_{2, i}(r)}{\pi d_{i}^{2}}, \\
\phi_{1, i}(r)=\frac{\phi_{2, i}(r)}{2 \pi d_{i}}, \\
\phi_{2, i}(z)=\frac{d_{i}^{2}}{4} \int_{0}^{2 \pi} d \varphi \int_{0}^{\pi} d \theta\left[\phi_{2}\left(r^{\prime}\right) \sin \theta\right],
\end{gathered}
$$

where $r^{\prime 2}=r^{2}+r d_{i} \cos \theta+\frac{d_{i}^{2}}{4}-\frac{d_{i}^{2}}{4} \sin ^{2} \theta \cos ^{2} \varphi$,

$$
\phi_{3, i}(r)=\pi \int_{0}^{d_{i} / 2} r_{1}^{2} d r_{1} \int_{0}^{2 \pi} d \varphi \int_{0}^{\pi} d \theta\left[\phi_{i}\left(r^{\prime}\right) \sin \theta\right]
$$


where $r^{\prime 2}=r^{2}+2 r r_{1}^{2} \cos \theta+r_{1}^{2}-r_{1}^{2} \sin ^{2} \theta \cos ^{2} \varphi$,

$$
\phi_{v 1, i}(r)=\frac{\phi_{v 2, i}(r)}{2 \pi d_{i}}
$$

and

$$
\phi_{v 2, i}(r)=-\frac{\mathbf{r}}{r} \frac{d_{i}^{2}}{4} \int_{0}^{2 \pi} d \varphi \int_{0}^{\pi} d \theta\left[\phi_{i}\left(r^{\prime}\right) \sin \theta \cos \theta\right] .
$$

As described in our previous work $[16,17]$, the reduced excess chemical potential due to the electrostatic potential is given by

$$
\beta \mu_{i}^{E L}(\mathbf{r})=\beta \mu_{i}^{E L}\left(\rho_{i}^{0}\right)-\sum_{j=+,-} \int \Delta \rho_{j}\left(\mathbf{r}^{\prime}\right) c_{i j}^{E L}\left(\left|\mathbf{r}-\mathbf{r}^{\prime}\right|\right) d \mathbf{r}^{\prime},
$$

where $c_{i j}^{E L}(t)$ is the direct correlation function (DCF). The mean spherical approximation (MSA) gives accurate analytic expressions for the $c_{i j}^{E L}(t)$. The result $[18,19]$ is

$$
c_{i j}^{E L}(t)=-2 l_{B}\left[-Z_{i} N_{j}+X_{i}\left(N_{i}+\Pi X_{i}\right)-\left(d_{i} / 3\right)\left(N_{i}+\Pi X_{i}\right)^{2}\right],
$$

for the range $0 \leq t \leq\left|d^{i}-d_{j}\right| / 2$ and

$$
c_{i j}^{E L}(t)=l_{B}\left[\left(d_{i}-d_{j}\right) L_{1}-t L_{2}+t^{2} L_{3}+t^{4} L_{4}\right],
$$

for the range $\left|d_{i}-d_{j}\right| / 2 \leq t \leq\left(d_{i}+d_{j}\right) / 2$. The MSA parameters are calculated from

$$
\begin{aligned}
L_{1}= & \frac{X_{i}+X_{j}}{4}\left(S_{i}-S_{j}\right)-\frac{d_{i}-d_{j}}{16}\left[\left(S_{i}+S_{j}\right)^{2}-4 N_{i} N_{j}\right], \\
L_{2}= & \left(X_{i}-X_{j}\right)\left(N_{i}-N_{j}\right)+\left(X_{i}^{2}+X_{j}^{2}\right) \Pi+\left(d_{i}+d_{j}\right) N_{i} N_{j} \\
& +\left[d_{i} S_{i}{ }^{2}+d_{j} S_{j}{ }^{2}\right] / 3, \\
L_{3}= & \frac{X_{i}}{d_{i}} S_{i}+\frac{X_{j}}{d_{j}} S_{j}+N_{i} N_{j}-\left[S_{i}{ }^{2}+S_{j}{ }^{2}\right] / 2, \\
L_{4}= & \frac{S_{i}^{2}}{6 d_{i}{ }^{2}}+\frac{S_{j}^{2}}{6 d_{j}{ }^{2}}
\end{aligned}
$$

with

$$
\begin{gathered}
S_{i}=N_{i}+\Pi X_{i} \\
\Pi=\left(\pi l_{B} \sum_{i} \rho_{i}{ }^{0} X_{i}{ }^{2}\right)^{1 / 2}
\end{gathered}
$$


and

$$
N_{i}=\frac{X_{i}-Z_{i}}{d_{i}}
$$

where $X_{i}$ is obtained from the nonlinear equation

$$
\left(1+\Pi d_{i}\right) X_{i}+\alpha d_{i}^{2} \sum_{j} \rho_{i}^{0} d_{j} X_{j}=Z_{i}
$$

where

$$
\alpha \equiv(\pi / 2)\left[1-(\pi / 6) \sum_{i} \rho_{i}{ }^{0} d_{i}^{3}\right]^{-1}
$$

It was shown in our previous work [20] that a quadratic expression is sufficient to capture such counter-intuitive phenomena as the attraction between like charges and charge inversion in the presence of multivalent ions that are not predicted by mean-field theories.

\section{Results}

Our DFT for cylindrical geometry was first tested by comparison with the MC results of Jamnik and Vlachy [21] for an aqueous electrolyte in a cylindical pore (Fig. 2). For this test case, the diameters of the cation and anion are both $d=0.42 \mathrm{~nm}$, the radius of the cylindrical pore is $a=2 \mathrm{~nm}$, the concentration of the reservoir ions is $\rho_{i}^{0}=0.1099 \mathrm{M}$, and the charge density of the cylindrical wall is $Q=0.0712 \mathrm{C} / \mathrm{m}^{2}$. We can see from Fig. 2 that the pore is mostly occupied by counterions. The density of the counterions decreases as the distance from the wall increases (i.e. $r$ decreases), whereas the density of the coions show reverse trend. The agreement between our DFT and MC is excellent, much better than that between the Poisson-Boltzmann equation and the simulation results. More extensive comparison of the DFT and MC data has been reported by Peng and Yu [22]. Hence, we are confident in our version of DFT for applications to ions in a cylindrical pore.

We have calculated $Q$ for a given set of $\psi_{0}$ and, using Eq. (9), obtained the differential capacitance, $C_{d}$. Results for $C_{d}$ are given in Fig. 3 for two bulk ionic concentrations, $\left(\rho_{i}^{0}=1.0 \mathrm{M}\right.$ and $\left.4.8 \mathrm{M}\right)$, and three values of $D=2 a(D=0.6 \mathrm{~nm}, 0.8 \mathrm{~nm}$, and $1.0 \mathrm{~nm})$. In this calculation and comparison, $d_{+}=d_{-}=0.5 \mathrm{~nm}$. At a low bulk concentration (Fig. $3(\mathrm{~b}))$, the $C_{d}-\psi_{0}$ curve shows a two-hump shape. With the increase of the pore diameter, the maximum value of $C_{d}$ also increases, while the shape of the $C_{d}-\psi_{0}$ curve shows a 

bulk concentration (Fig. 3(a)), the $C_{d}-\psi_{0}$ curve shows a one-hump shape instead of a two-hump shape. With an increase of the pore diameter, the maximum value of the $C_{d}$ also increases. Our calculations clearly show a continuous transition from a two-hump shape at low concentrations to a single-hump or bell-curve shape at higher concentrations. At the lower concentration, the two humps merge into a single hump as $D$ increases. This is similar to what has been observed for a single surface $[6,7,8]$ and for a slit pore [23, 24]. Additionally, we observe that, when there are two humps, the maximum value of $C_{d}$ decreases with increasing $D$ in both our calculation and that of Kornyshev [5]. However, in his study the separation of the two humps increases with increasing $D$ whereas the opposite behavior is seen in our DFT calculation.

The volume averages of the amount of ion adsorption, defined by

$$
\Gamma_{i}=\frac{2}{a^{2}} \int_{0}^{a} \rho_{i}(r) r d r
$$

and $\Gamma=\Gamma_{+}+\Gamma_{-}$are plotted in Fig. 4. At lower values of the surface potential, the increase of the charge in the channel results from a depletion of the counterions and attraction of the counterions whereas at higher values of the surface potential, the increase of the charge in the channel is mainly due to the increase of the counterions. For the case of single-hump curve (Fig.3(a) and Fig.4(a)), after the counterions are totally depleted $\left(\left|\psi_{0}\right| \approx 0.2 \mathrm{~V}\right)$, the decrease of $C_{d}$ slows down, and begins to follow a trend of $C_{d} \propto\left|\psi_{0}\right|^{-1 / 2}$. This is common for the change of $C_{d}$ with $\psi_{0}$ when all the coions are depleted and seems to be independent of the model used [25]. For the case of two-hump curve (Fig.3(b) and Fig.4(b)), when the counterions are totally depeted $\left(\left|\psi_{0}\right| \approx 0.1 \mathrm{~V}\right)$, the $C_{d}$ value in the $C_{d}-\psi_{0}$ curve shows a maximum. This indicates that the adsorption behavior of the ions in the channel has important effects on the $C_{d}-\psi_{0}$ curve.

We also considered the influences of ion asymmetry on the ionic distributions and the capacitances. Fig. 5(a) gives the $C_{d}-\psi_{0}$ curve for the case of $d_{+}=0.5 \mathrm{~nm}$ and $d_{-}=0.6$ $\mathrm{nm}$ with three different pore diameters $(D=0.7 \mathrm{~nm}, 0.9 \mathrm{~nm}$, and $1.1 \mathrm{~nm})$. Here the bulk concentration is $\rho_{i}^{0}=1.0 \mathrm{M}$. We can see that, as was the case for symmetric ions, there is a continuous transition from two-hump shape curve to one-hump curve with the increase of the pore diameter. The maximum point is located at a negative $\psi_{0}$, this is clearly due to the smaller size of the cations. Fig. 5(b) gives the adsorptions of cations, anions, and total ions for the case with $D=0.9 \mathrm{~nm}$. It also shows that the depletion point of the ions corresponds to the point of maximum in the $C_{d}-\psi_{0}$ curve. 


\section{Summary}

Motivated by the intriguing paper of Kronyshev [5], density functional theory has been applied to study the differential capacitance of a model organic electrolyte (modeled as charged hard spheres in a dielectric continuum) in a cylindrical pore with a hard surface. The cylinder is so narrow that the ions can form only a single file. The model is intended to mimick an organic electrolyte. Solvent molecules are represented as a dielectric continuum and not explicitly considered in the model electrolyte that we employ. We find that the differential capacitance has a double hump at low ionic concentrations but that as the ionic concentration is increased, the minimum between the humps fills in and the differential capacitance acquires a bell shape or single hump. The continuous transition to a single hump occurs at lower concentrations as the cylinder diameter is narrowed. This is consistent with previous findings for other geometries but contrasts with Kornyshev's result [5], who finds only a double hump capacitance shape. Of course, his observation is valid only for the parameters that he employs but his results show no tendency to form a single hump. Kornyshev considered a cylinder with a metallic surface and included the contributions of electrostatic images $[5,26]$. In contrast, our cylinder surface has the same dielectric constant as the cylinder interior and no images are present. However, to obtain his results, Kornyshev used an image interaction potential that was obtained by considering ions only at the center of the cylinder. He used an imaginative Ising-like approximation but its accuracy is uncertain. As a result, it is unclear whether the persistent double hump capacitance curves that he finds is due to metallic images or the approximations that he used. We plan future simulations and modified Poisson-Boltzmann theory calculations to shed more light on this question.

\section{Acknowledgements}

The authors are grateful for the comments of Alexei Kornyshev concerning his work. This work was supported as part of the Fluid Interface Reactions, Structures and Transport (FIRST) Center, an Energy Frontier Research Center funded by the U.S. Department of Energy, Office of Science, Office of Basic Energy Sciences. X. K. is grateful to the Chinese Scholarship Council for a visiting fellowship. The numerical calculations were performed at the National Energy Research Scientific Computing Center (NERSC). 


\section{References}

[1] A. Yu, Electrochemical supercapacitors for energy storage and delivery: fundamentals and applications, Taylor \& Francis, Boca Raton, 2013.

[2] P. Simon and Y. Gogotsi, Nat. Mater. 7 (2008) 845-854.

[3] J. Chmiola, G. Yushin, Y. Gogotsi, C. Portet, P. Simon and P. Taberna, Science, 313(2006) 1760-1763.

[4] C. Largeot, C. Portet, J. Chmiola, P. Taberna, Y. Gogotsi and P. Simon, J. Am. Chem. Soc. 130(2008) 2730-2731.

[5] A. A. Kornyshev, Faraday Discuss. 164 (2013) 117-133.

[6] S. Lamperski, C. W. Outhwaite, and L. B. Bhuiyan, J. Phys. Chem. B, 113 (2009) 8925-8929.

[7] S. Lamperski and D. Henderson, Mol. Sim. (2011) 264-268.

[8] D. Henderson, S. Lamperski, Z. Jin, and J. Wu, J. Phys. Chem. B, 115 (2011) 1291112914

[9] D. Gillespie, J. Phys. Chem. Lett., 2 (2011) 1178-1182.

[10] C. W. Outhwaite, L. B. Bhuiyan, Mol. Phys., 74 (1991) 367-381.

[11] L. B. Bhuiyan, S. Lamperski, J. Wu and D. Henderson, J. Phys. Chem. B, 116 (2012) 10364-10370.

[12] J. Wu, T. Jiang, D. Jiang, Z. Jin and D. Henderson, Soft Matter, 7 (2011) 11222-111231.

[13] Y. X. Yu and J. Z. Wu, J. Chem. Phys. 117 (2002) 10156-10164.

[14] Z. D. Li and J. Z. Wu, Phys. Rev. E 70 (2004) 031109.

[15] Y. Rosenfeld, Phys. Rev. Letters 63 (1989) 980-983.

[16] J. Jiang, D. P. Cao, D. Henderson, and J. Z. Wu, J. Chem. Phys. 140 (2014) 044714.

[17] J. Jiang, D. P. Cao, D. Henderson, and J. Z. Wu, Phys. Chem. Chem. Phys. 16 (2014) 3934-3938. 
[18] L. Blum, Mol. Phys., 30 (1975) 1529-1535.

[19] K. Hiroike, Mol. Phys., 33 (1977) 1195-1198.

[20] Z. D. Li and J. Z. Wu, J. Phys. Chem. B 110 (2006) 7473-7484.

[21] B. Jamnik and V. Vlachy, J. Am. Chem. Soc. 115 (1993) 660-666.

[22] B. Peng and Y.X. Yu, J. Chem. Phys. 131 (2009) 1347031-1347037.

[23] A. A. Kornyshev, J. Phys. Chem. B 111 (2007) 5545-5557.

[24] A. A. Lee, S. Kondrat, and A. A. Kornyshev, Phys. Rev. Lett. 113 (2014) 048701.

[25] M. V. Fedorov and A. A. Kornyshev, Chem. Rev. 114 (2014) 2978-3036.

[26] C. C. Rochester, A. A. Lee, G. Pruessner, and A. A. Kornyshev, Chem. Phys. Chem. 14 (2014) 4121-4125. 


\section{Figure Captions}

Figure 1 Single file ions in a cylindrical pore electrode. The pore is infinitely long in the axial direction and the fluid is in equilibrium with a reservior of ions at a specified constant concentration. The diameter of the cylinder is $D=2 a$ and ion diameters and charges of the cations and anions are $d_{+}, d_{-}, Z^{+} e$, and $Z^{-} e$, respectively. The surface of the cylinder is held at a constant electrical potential, $\psi_{0}$.

Figure 2 Validation of our DFT calculation by comparison with MC data taken from reference 21. The diameter of the ions is $d=042 \mathrm{~nm}$, the radius of the channel is $a=2 \mathrm{~nm}$, and the surface charge density is $Q=0.0712 \mathrm{C} / \mathrm{m}^{2}[21]$ The circles and curves give the MC results and the results of our DFT calculation, respectively.

Figure 3 Differential capacitance, $C_{d}=\partial Q / \partial \psi_{0}$ as a function of the potential of the cylinder surface, $\psi_{0}$, for two electrolyte concentrations and three values of the cylinder nanopore diameter, $D=2 a$.

Figure 4 Volume averaged adsorption amount, $\Gamma$ as a function of the potential of the cylinder surface, $\psi_{0}$, for two electrolyte concentrations and for $D=2 a=1 \mathrm{~nm}$.

Figure 5 (Left panel) Differential capacitance as a function of the potential of the cylinder surface, $\psi_{0}$, for the lower concentration $(1 \mathrm{M})$ and three values of $D$. The diameters of ions are $d^{+}=0.5 \mathrm{~nm}$ and $d^{-}=0.6 \mathrm{~nm}$. (Right panel) Adsorption as a function of the potential of the cylinder surface, $\psi_{0}$ for the case of $D=0.9 \mathrm{~nm}$. 


\section{Graphical Abstract (for review)}

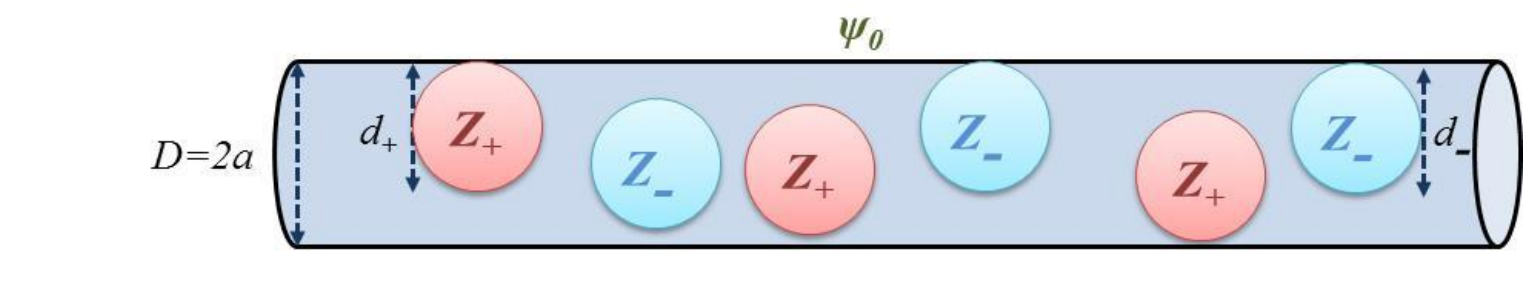

. 


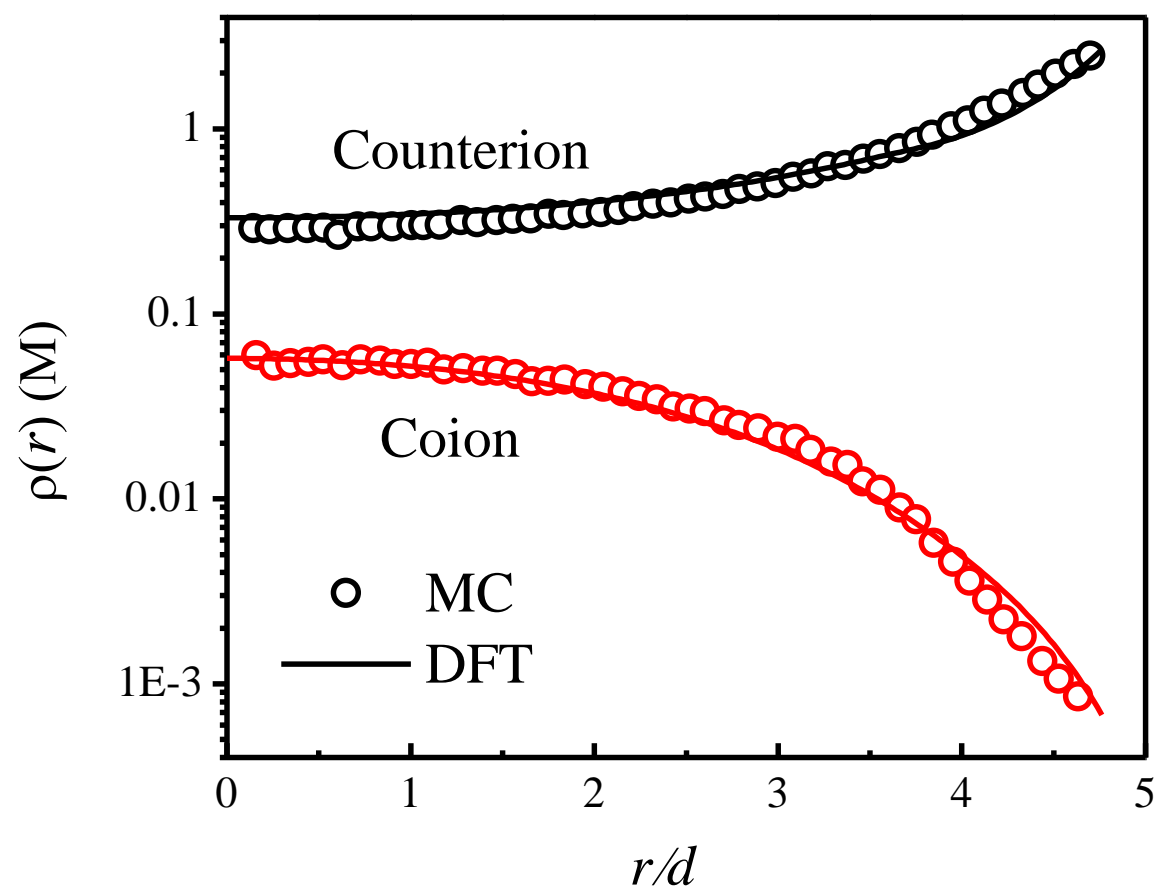




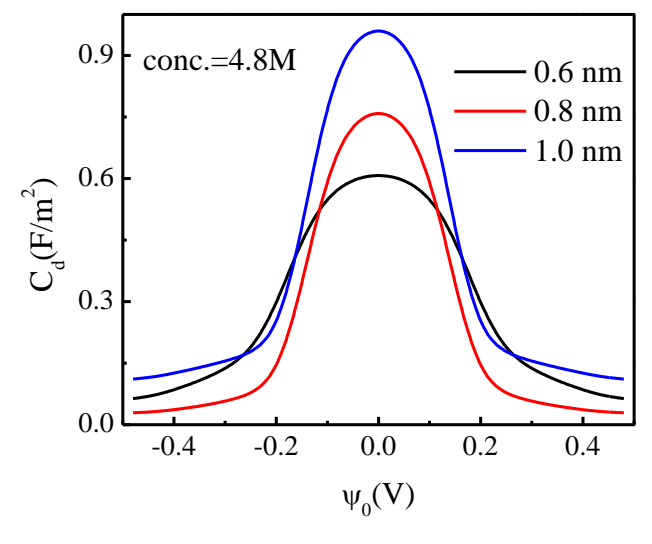

a

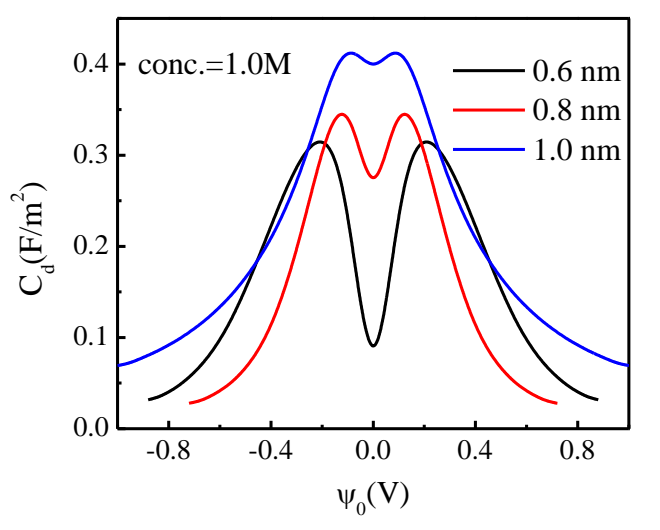

b

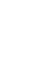




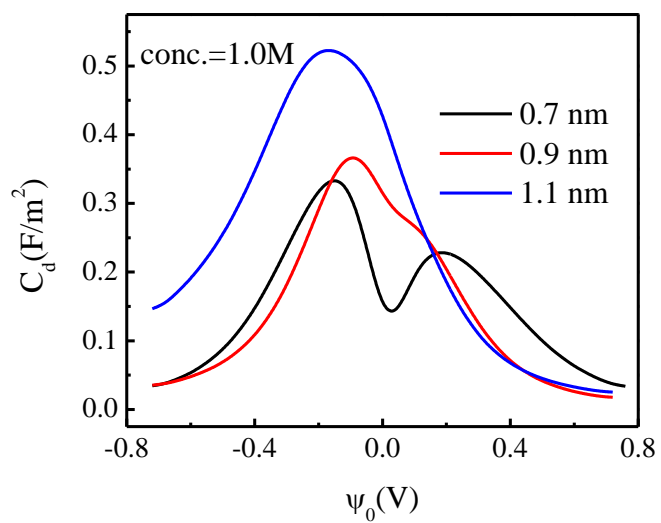

a

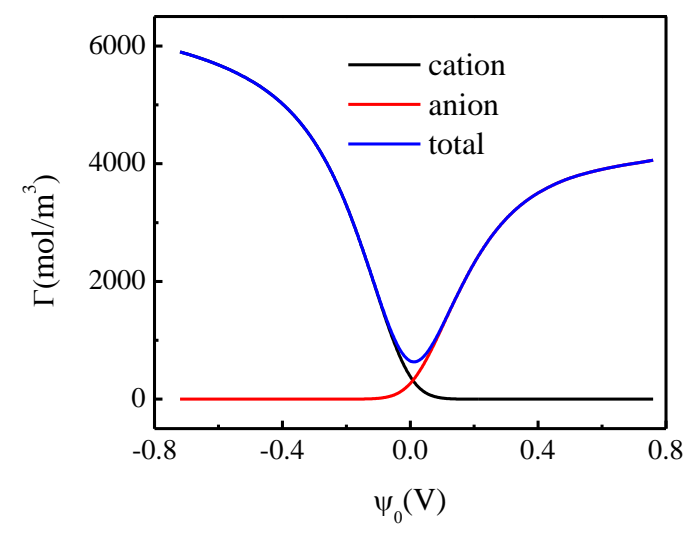

b

\section{列}

\footnotetext{
$+2$
}$$
\text { . }
$$

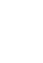

